\title{
THE CHRISTMAS MEETING IN CHICAGO
}

The two hundred sixty-sixth, thirty-first regular Western, meeting of the Society was held at the University of Chicago on Monday, December 31, 1928. About sixty persons attended the meeting, among whom were the following fifty members of the Society:

R. W. Babcock, Bartky, Beenken, O. E. Brown, L. H. Bunyan, G. N. Carmichael, R. D. Carmichael, Chittenden, A. G. Clark, H. H. Conwell, C. F. Craig, Crathorne, Curtiss, Dickson, Escott, Everett, Feltges, Gouwens, L. M. Graves, Griffiths, V. G. Grove, Holgate, Ingraham, B. W. Jones, B. F. Kimball, E. P. Lane, Langer, LaPaz, Leavens, Logsdon, McCoy, March, Marshall, E. H. Moore, T. W. Moore, Pepper, Pettit, H. L. Rietz, Ross, Roth, Sanger, Schottenfels, Simmons, Wall, Warren Weaver, Weiss, F. E. Wood, Frederick Wood, Roscoe Woods, Worth.

In the evening thirty-seven members and guests attended dinner in the Del Prado Hotel. Professor Lane acted as toastmaster and, before the dinner, called upon Professor E. H. Moore, who could not remain for the dinner. Professor Moore spoke for a few minutes, welcoming the members and guests to Chicago. After the dinner the toastmaster called upon Professor R. D. Carmichael, who told of the New York meeting, Professor Chittenden, who talked of the coming Des Moines meeting, Professor Ingraham, who spoke of the problems of the location of meetings in the MidWest and suggestions that had been made towards increasing the interest of the programs, and Miss Weiss, who spoke for the ladies.

The papers whose abstracts appear below were read as follows: papers 1-9 in the morning, and papers 10-19 in the afternoon. Papers $6,8,9$ and $15-19$ were read by title, the rest in person. Professor Marshall presided in the morning and Professor Curtiss in the afternoon.

1. Dr. B. W. Jones: The number of representations by certain positive ternary quadratic forms.

The number of solutions of $x^{2}+y^{2}+z^{2}=n$ in integers for any positive integer $n$ is a known function of the class number of $n$ But the number of 
integral solutions of $a x^{2}+b y^{n}+c z^{2}=n$, where $a, b$ and $c$ are positive integers, is not, in general, known. In this paper, by means of a transformation taking the form $x^{2}+2 y^{2}+3 z^{2}$ into $x^{2}+y^{2}+z^{2}$, the number of representations of $n$ by the former is shown to be a simple function of the number of representations by the latter of $6 n$ and, in some cases, other multiples of $n$. From these results can be found the number of representations of $n$ by the former in terms of the class numbers of certain multiples of $n$. This type of relationship has been considered only incidentally by Nazimoff and Uspensky and only fragmentary results have been obtained for this form. It is demonstrated that not only the form mentioned but many other new forms, seemingly unrelated to the sum of three squares, yield to similar treatment.

2. Dr. Lois W. Griffiths: A generalization of the theorem of Fermat and Cauchy on polygonal numbers.

This theorem, that every positive integer is the sum of $m+2$ polygonal numbers of order $m+2$ and that all but four of these may be taken to be 0 or 1 , has been extensively generalized by Dickson. Another generalization is suggested by the following restatement of the theorem. Let $n, a_{1}, \cdots, a_{n}$ be positive integers and $p_{1}, \cdots, p_{n}$ be polygonal numbers; then the function $f=a_{1} p_{1}+\cdots+a_{n} p_{n}$ is universal when $n=m+2$ and $a_{1}=\cdots=a_{n}=1$, and $p_{5}, \cdots, p_{m+2}$ may be taken to be 0 or 1 if $m>2$. In this paper the universal functions $f$ are determined for $m \geqq 3$, $a_{1}+\cdots+a_{n} \leqq m+2$; the cases $m=1$ and 2 have been treated for any $n$ by Liouville and Dickson.

3. Professor L. E. Dickson: All universal ternary quadratic forms are null forms.

A positive or indefinite form $f$ is called universal if it represents all positive integers or all positive and negative integers respectively. For threevariablesevery universal $f$ is proved to be a null form, that is, $f$ vanishes for integral values not all zero of the variables. Since all universal Null ternary forms were found in another paper presented by the writer at this meeting, this completes the theory of all universal ternary quadratic forms.

4. Professor L. E. Dickson: All indefinite quadratic forms in 3 or more variables which represent every integer.

Of ternary quadratic forms, no positive form represents every positive integer, but infinitely many indefinite forms are universal, that is, represent every positive, negative, and zero integer. All universal $a x^{2}+b y^{2}+c z^{2}$ are determined explicitly. A method is developed to find all Null universal quadratic forms in $n$ variables. The results are complete for $n=3$ and essentially complete for $n=4$. For $n \geqq 5$, the theory is sufficient but involves more computation.

5. Professor F. E. Wood: Cubics whose (Hessian) ${ }^{n}$ are themselves.

This paper appears in full in the present issue of this Bulletin. 


\section{Professor K. E. Rosinger: A note concerning the sym-} bols $\phi x$ and $\phi \hat{x}$.

It is the purpose of this paper to examine the basis of the distinction made in Principia Mathematica between the symbols $\phi x$ and $\phi \hat{x}$. The problem is considered in the light of Frege's studies in the logical analysis of functions. It is found that the distinguishing characteristic of propositional functions recognized by Whitehead and Russell, namely "ambiguity," is derived from a more fundamental notion, which Frege calle Ergänzungsbedürftigkeit. This notion is represented by Principia's symbol $\phi x$. On the other hand, $\phi \hat{x}$ expresses Frege's concept Zusammengehörigkeit. It appears that neither Frege nor Principia have recognized that these two concepts are independent of each other. Hence the distinction between $\phi x$ and $\phi \hat{x}$ is fundamental. The fact that this has not been detected by many mathematical logicians has produced considerable confusion. From this fundamental difference, the paper deduces a number of properties of the notions here considered, thereby bringing to explicit statement the meanings and purposes of $\phi x$ and $\phi \hat{x}$.

\section{Dr. H. S. Wall: On extended Stieltjes series.}

This paper refers to the author's previous article which appears in the Transactions of this Society, January 1929, on Stieltjes series and continued fractions. The writer considers the problems of extending the Stieltjes series

$$
\sum_{i=0}^{i=\infty} c_{i}(-z)^{i}
$$

to the left by prefixing successively terms of the form $(-1)^{k} c_{-k} z^{-k}, k=1,2$, $3, \cdots, n$, in such a way that all of the series

$$
\sum_{r=0}^{r=\infty} c_{-k+r}(-z)^{r},(k=1,2,3, \cdots, n),
$$

shall be Stieltjes series. A necessary and sufficient condition for a first extension, given in the preceding article, is replaced by the following. Let the limit of the sequence of even convergents of the Stieltjes continued fraction corresponding to (1) be

$$
F(z)=\int_{0}^{\infty} \frac{d \phi_{1}(u)}{1+z u}
$$

Then (1) admits a first extension if and only if

$$
\lim _{a \rightarrow 0} \int_{a}^{\infty} \frac{d \phi_{1}(u)}{u},(a>0)
$$

exists and has a finite value $k$; and $c_{-1} \geqq k$. From this it follows that for a large class of Stieltjes series, including among others those for which the corresponding Stieltjes continued fractions are divergent, extensions of infinite order exist in which

$$
c_{-k}=\int_{0}^{\infty} \frac{d \phi_{1}(u)}{u^{k}} .
$$


The Stieltjes continued fraction corresponding to the Stieltjes series

$$
\sum_{i=1}^{i=\infty}(-1)^{i+1} \frac{C_{i}}{z^{i}}
$$

converges for all $z$ not belonging to the interval $(0,-R)$, where $R$ is the distance from the origin to the most remote pole of (2), to $F(z)$, and the series (3) converges for all $|z|>R$ to the function $F(z)$.

\section{Professor R. L. Wilder: A topological definition of simple closed surface.}

The following characterization of simple closed surface is obtained. In a metric separable space let $M$ be a compact set containing at least one simple closed curve, and satisfying the following conditions: (1) If $t$ is an arc of $M$, then $M-t$ is connected; (2) if $J$ is a simple closed curve of $M$, then $M-J$ is the sum of two uniformly regular components. Then $M$ is homeomorphic with the sphere $x^{2}+y^{2}+z^{2}=1$.

\section{Professor R. L. Wilder: Concerning the Phragmen-} Brouwer Theorem.

Let $S$ be a topological space. Consider the following properties: I. If $F_{1}$ and $F_{2}$ are mutually exclusive closed subsets of $S$, neither of which separates $S$, then $F_{1}+F_{2}$ does not separate $S$. II. If $N$ is a continuum in $S$ and $R$ a component of $S-N$, the boundary of $R$ is a continuum. Either of these properties serves as a statement of the Phragmen-Brouwer Theorem if $S$ is a euclidean space. It is the purpose of the present paper to study the equivalence between these properties, and certain related properties, as well as to determine certain spaces in which they hold true. Examples are given of spaces where I holds but II does not hold, and conversely. It is shown, however, that in a certain class of general spaces, of which euclidean spaces form a proper subset, the two properties are equivalent. The following generalization of the theorem of Kuratowski (Fundamenta Mathematicae, vol. 8, p.149) is obtained: If, in a euclidean plane $E_{2}, M$ is a bounded continuum containing more than one prime part and such that no one of its prime parts separates $E_{2}$, then in order that $M$ may be considered as a space having property II it is necessary and sufficient that $M$ should not separate $E_{2}$.

10. Professor V. G. Grove: A canonical form of Green's projective analogue of the Gauss differential equations.

In this paper the author develops a canonical form of the projective analogue of the Gauss differential equations. The vertices of the tetrahedron of reference are covariants of simple geometric significance. Each invariant appearing in the canonical form has a geometric significance easily read from the equations. By specializing the parametric net to be conjugate, one obtains the equivalent of Lane's canonical form for conjugate nets. By assuming the parametric net to be non-conjugate, a canonical 
form for Green's differential equations of a non-conjugate net is obtained. In this case also the geometrical significance of the invariant coefficients may be seen directly from the differential equations.

11. Professor E. P. Lane: On the fundamental transformation of surfaces.

The author develops the theory of a fundamental transformation of surfaces in ordinary space, treating the subject projectively and using Wilczynski's method based on a completely integrable system of differential equations. The conjugate and harmonic invariants recently used by Slotnick receive new geometric interpretations. Certain quadric surfaces called the associated quadrics of the transformation are introduced into the theory. Various theorems are obtained concerning fundamental transformations with conjugate and harmonic congruences of special types.

12. Professor R. E. Langer: On the asymptotic forms of the solutions of certain differential equations containing a parameter.

This paper deals with the equation $y^{\prime \prime}+\lambda^{2} \phi(x) y=0$ in which $\phi(x)$ vanishes like $x^{k}$ at $x=0$. It is shown that a certain approximate equation is solvable in terms of Bessel's functions, and that there exists in any upper or lower half $\lambda$-plane a pair of solutions of the given equation which for $0<\alpha \leqq x \leqq \beta$ have asymptotic forms with the same leading terms as the solutions of the approximate equation, and which are also represented asymptotically by the latter at $x=0$. By means of these solutions and a formula expressing the general solution $y(x)$ in terms of an arbitrary pair $y_{1}(x) y_{2}(x)$, one obtains the leading terms of the asymptotic expressions representing $y(x)$ for $\lambda$ in different half-planes, or $x$ in different intervals on opposite sides of the origin.

\section{Professor L. E. Dickson: New results and methods for} minima of quadratic forms.

In 1903 Markoff obtained the first three minima of all real indefinite ternary quadratic forms for integral values of the variables, and emphasized the difficulty of the problem of finding further minima. The fourth mimimum is now found by his method. But new methods are developed to obtain further minima. An application is to the tabulation of representatives of all classes of a given determinant. Such a table is used to obtain general theorems on the representation of all integers by such quadratic forms.

14. Professor M. H. Ingraham: The reducibility of algebras without a finite base.

Systems are considered which satisfy the usual postulates for a linear associative algebra except they may not contain a finite base. From the definition of a linear set as one which is closed under addition and scalar 
multiplication, we find that the definition of the sum of a class of linear sets, as the minimal linear set containing every linear set of the class, has content. As Wedderburn has shown under slightly stronger hypotheses, most of the elementary theorems on the direct sum of linear sets follow with, at most, a slight change in the usual proof. Algebras exist containing no irreducible sub-algebras. If an algebra can be reduced into irreducible sub-algebras, each with a principal unit, the reduction apart from order is unique. An algebra with a principal unit cannot be expressed as the direct sum of more than a finite number of algebras. This last fact is partly due to restrictions in the definition of a sum rather than being inherent in the problem.

\section{Professor E. T. Bell: A partial isomorphism between} the functions of Lucas and Weierstrass.

This paper will appear in full in an early issue of this Bulletin.

16. Professor E. T. Bell: A class of polynomials and rational functions in four variables.

The polynomials and functions are those arising as coefficients o $1, i, j, k$ in the $n$th power, $n$ an integer, of the quaternion whose coordinates are $w, x, y, z$. These functions have a number of interesting properties, of which the following will suffice as specimens. With respect to one and only one of the independent variables, the polynomials are Appell polynomials. With respect to a properly chosen argument, the polynomials are Tchebycheff polynomials. With respect to $n$, both the polynomials and the functions are Lucas functions. Both the polynomials and the functions, with respect to $w, x, y, z$, satisfy an extremely simple set of 4 self-adjoint differential equations of the second order. The polynomials are rationally resolvable into factors of degrees $\leqq 2$ in an elegant way. With respect to $n$ they have addition and subtraction theorems abstractly identical with those for the circular, hyperbolic and Lucas functions. If the norm of the generating quaternion is unity, the polynomials and functions, in their algebraic aspects, are simply isomorphic with the sigma, zeta, and pe functions of Weierstrass.

17. Professor E. T. Bell: A class of polynomials and rational functions in eight variables.

These are the generalization of the preceding four-variable polynomials and functions to the like arising from the eight-square theorem of Degen and Cayley. The preceding abstract may be repeated verbatim with respect to these. No further generalization in the same direction is possible.

\section{Professor W. A. Manning: The degree and class of doubly transitive groups.}

In this paper it is proved that if $\mu$ is the class and $n$ the degree of a doubly transitive group which contains a substitution of degree $\mu$ and of 
prime order $p(>3)$, then $\mu>(n / 2)(1-1 / p)-\left(n^{1 / 2} / 2\right)\left(1-1 / p^{2}\right)^{1 / 2}-1$. The same limit holds when $p=3(\mu>3)$ if $n$ is sufficiently large. This result complements the limit $\mu>n / 2-n^{1 / 2} / 2-1$, for $p=2$, published by the author in 1914.

19. Professor T. H. Hildebrandt: Remarks on Carathéodory's theory of measure.

Carathéodory bases his theory of measure on an upper measure function satisfying five postulates. This paper shows how from an upper measure function satisfying the first three postulates it is possible to construct an upper measure function satisfying the fifth, and obtains conditions under which the property of measurability is unaltered. The same study is undertaken beginning with an upper measure function satisfying only the first postulate.

\section{H. INGRAHAM, Associate Secretary.}

\section{A CORRECTION}

BY W. J. TRJITZINSKY

In my paper, Zeros of a function and of its derivative, (this Bulletin, Vol. 33 (1927), pp. 693-695), $2 \pi$ should be replaced everywhere by $2 \pi n$ (integer $\geqq 1$ ), except on line 7 , page 693 , and in expressions $1 /(2 \pi i)$. 